\title{
Venous thromboembolic disease and pregnancy
}

\author{
Luis J. Marfil-Rivera* \\ Department of Internal Medicine, University Hospital “Dr. Jose E González,"Autonomous University of Nuevo Leon, Monterrey, Nuevo Leon, Mexico
}

\begin{abstract}
The incidence of venous thromboembolism (VTE) during pregnancy is increased by 4-50 times. It is estimated that VTE complicates between 1 and 1.5 per 1000 pregnancies. During pregnancy, hemostasis is characterized by a prothrombotic state to prevent possible bleeding during childbirth and postpartum; however, this change predisposes the mother to an increased risk of VTE. The diagnosis of VTE in pregnancy is a challenge because the clinical findings of this disease may be confused by the physiological changes of pregnancy. The treatment of VTE requires the use of anticoagulants drugs and in the pregnant patient is required a more controlled protocol. For this reason, it is important to know the physiological changes during pregnancy and the pharmacodynamics of anticoagulant drugs to prevent any hemorrhagic complications during childbirth.
\end{abstract}

Key words: Pregnancy. Venous thrombosis. Anticoagulant therapy. Blood coagulation.

\section{Introduction}

Of the possible emergencies which could occur during pregnancy, venous thromboembolism (VTE) might be the most critical; therefore, it is necessary to know the changes which develop during pregnancy and post-partum to identify those patients with a high risk of thrombosis, perform a timely diagnosis, and begin proper treatment for each case. During pregnancy, a prothrombotic stage is generated to prevent a possible hemorrhage during implantation, placentation, or delivery; however, these changes increase the risk of thrombosis $^{1,3}$. Pregnancy, as a risk factor for thrombosis, increases its frequency by 5-10 times compared to non-pregnant women. Its prevalence is calculated to be between 0.76 and 1.7 events per 1000 pregnancies, managing to surpass hemorrhages and hypertension as the main cause of maternal morbidity-mortality ${ }^{1,4,5}$. The risk of thrombosis is uniform throughout the entire pregnancy, yet the greater risk is during the first 6 weeks' post-partum, with $80 \%$ of events occurring in the first 3 weeks' post-partum. C-sections increase the risk of thrombosis by between 5 and 9 times compared to vaginal delivery ${ }^{1,5-7}$.

VTE represents $14.9 \%$ of maternal deaths worldwide and in the western world is responsible for $10 \%$ of them (1.1 maternal deaths per 100,000 deliveries) $)^{8}$. Between $75 \%$ and $80 \%$ of cases occur as deep venous thrombosis (DVT), while between $20 \%$ and $25 \%$ are caused by pulmonary thromboembolism $(\mathrm{PTE})^{6}$. Along with acute episodes, there is an additional risk of venous insufficiency; $80 \%$ of women with DVT develop post-thrombotic syndrome, and up to $60 \%$ of patients develop venous insufficiency at 5 years of follow-up ${ }^{9}$. Estimations suggest that at least half the cases who develop DVT or a PTE during pregnancy suffer from subjacent thrombophilia. Hence, a complete knowledge of coagulation

\section{Correspondence:}

*Luis Javier Marfil-Rivera

E-mail: Imarfil@icloud.com
Available online: $27-05-2019$

Date of reception: 18-12-2018

Date of acceptance: 12-04-2019

DOI: 10.24875/RMU.19000043
Medicina Universitaria. 2019;21(1):29-33 www.medicinauniversitaria.org

México SA de CV. This is an open access article under the CC BY-NC-ND license (http://creativecommons.org/licenses/by-nc-nd/4.0/). 
Table 1. VTE risk factors

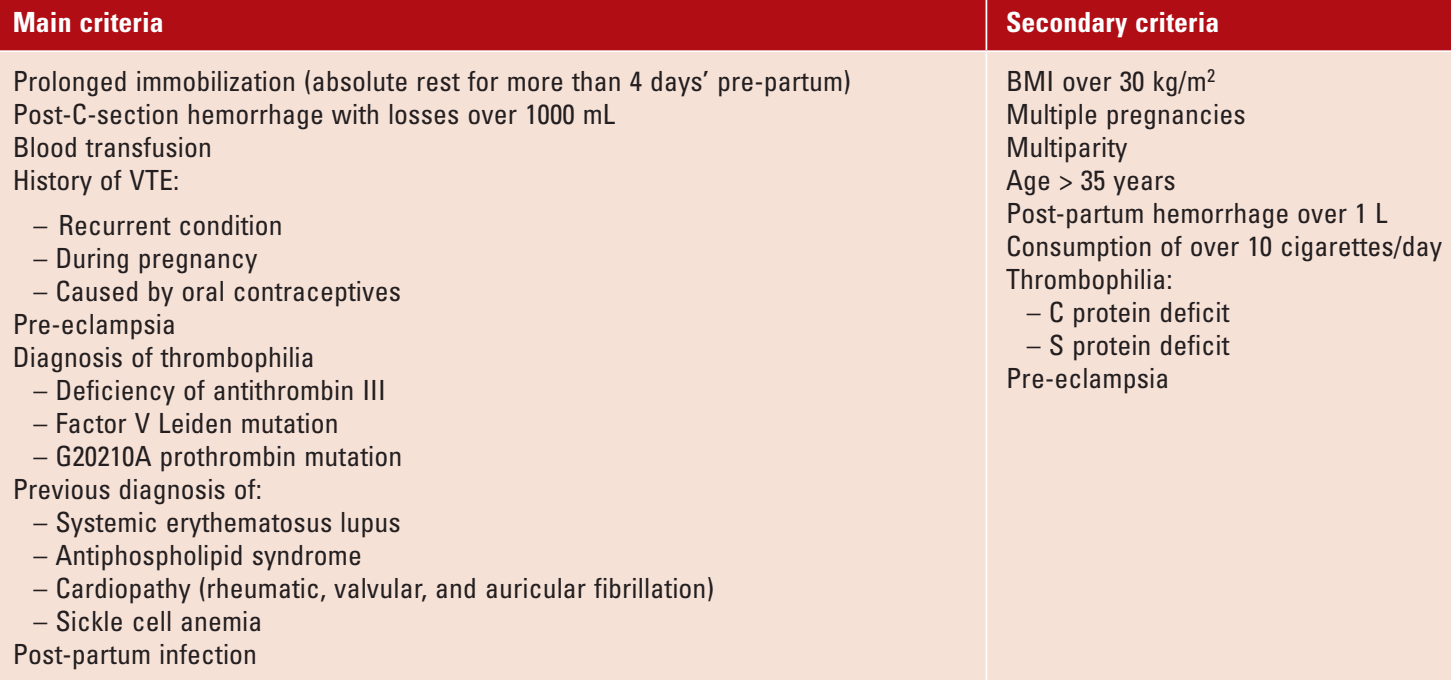

BMI over $30 \mathrm{~kg} / \mathrm{m}^{2}$

Multiple pregnancies

Multiparity

Age $>35$ years

Post-partum hemorrhage over $1 \mathrm{~L}$

Consumption of over 10 cigarettes/day

Thrombophilia:

- C protein deficit

- S protein deficit

Pre-eclampsia

systems is essential, as well as of fibrinolysis and its inhibitors in relation to pregnancy ${ }^{2,3}$. Diagnosis of thromboembolic events during pregnancy represents a challenge since clinical data are often masked by the physiological changes typical of gestation. There are several guidelines to identify risk factors linked to venous thromboembolic disease during pregnancy. In the following table, there is a classification which separates risk factors by the level of importance (Table 1).

Physiological changes which occur during pregnancy create a hypercoagulable state secondary to the increase of plasmatic factors II, VII, VIII, IX, X, and XII and an increase in the synthesis of fibrinogen. $S$ protein levels are lower, and even though $\mathrm{C}$ protein levels maintain stable, there is a resistance to the action of activated $C$ protein. On the other hand, the placenta produces inhibitors of the plasminogen activator, which alters fibrinolysis ${ }^{3}$.

Venous stasis occurs as a result of the vascular compression exerted by the gravid uterus and the vascular dilation mediated by estrogens. A reduction in flow speed linked to an increase in diameter of the lower limbs' veins has been proven, which is more noticeable during the last trimester.

Finally, even though pregnancy does not cause endothelial damage itself, delivery does, more so if instrumentation or a C-section are performed. Said damage might be more intense if the pregnant patient previously smoked 2,3 .

\section{Diagnosis DVT}

Most of these DVT cases during pregnancy occur in the lower limbs, with a predisposition for the left lower $\operatorname{limb}(90 \%)^{1,10}$.

The most common clinical data are acute onset of symptoms, erythema, pain, unilateral extremity heat and edema, lower abdominal pain, positive signs of hommas, and reflex arterial spasms, with cold and pale extremities, in addition to diminished pulses and difficulty with ambulation.

In the non-pregnant population, predictive clinical models such as the Wells score for DTV (Table 2) stratify patients to establish the possibility of having a thrombotic event, according to which they perform confirmatory imaging diagnosis. In the presence of a negative D-dimer (DD), the negative predictive value is $99.5 \%, 1,10$.

Additional studies include DD, which indicates a process of fibrinolysis subsequent to a thrombosis. Plasmatic levels are elevated in DVT and PTE, neoplasias, strokes, arterial thrombosis, disseminated intravascular coagulation, pneumonia, pregnancy, recent trauma, or hepatopathy. It increases during pregnancy, thus making it of little help, and its usefulness in pregnancy is still a topic of great debate ${ }^{9}$.

Imaging studies such as the Doppler ultrasonography are the standard method for the diagnosis of PVT. It is highly sensitive (92\%) and specific (98\%) for thrombosis ${ }^{11}$. Contrast venography and impedance plethysmography have fallen into disuse and are not 
Table 2. Wells score for deep venous thrombosis

\begin{tabular}{|l|c|}
\hline Clinical characteristics & Score \\
\hline $\begin{array}{l}\text { Active cancer (treatment within } 6 \text { months or in } \\
\text { palliative treatment) }\end{array}$ & +1 \\
\hline Paralysis, paresis, or lower extremity immobilization & +1 \\
\hline $\begin{array}{l}\text { Bed rest for more than } 3 \text { days as a result of } \\
\text { surgery (within } 4 \text { weeks) }\end{array}$ & +1 \\
\hline $\begin{array}{l}\text { Localized sensitivity throughout the venous system } \\
\text { distribution }\end{array}$ & +1 \\
\hline Edema in the entire leg & +1 \\
\hline Unilateral edema of the lower leg $>3$ cm & +1 \\
\hline Orange-skin edema only in the symptomatic leg & +1 \\
\hline Non-varicose collateral superficial veins & +1 \\
\hline Alternative diagnosis at least as probable as DVT & -2 \\
\hline Total score & \\
\hline
\end{tabular}

Interpretation of risk score (probability of DVT), $\geq 3$ points, high risk (75\%), 1-2 points, moderate risk $(17 \%),<1$ point, low risk (3\%), DVT: Deep venous thrombosis.

always available for the detection of $\mathrm{DVT}^{12}$. Magnetic resonance imaging (MRI) has a sensitivity between $94 \%$ and $96 \%$, with a specificity of $90-92 \%$ for the detection of DVT; however, the use of intravenous gadolinium during pregnancy remains a topic of debate. It goes through the placenta and is classified as a category-C drug by the Food and Drug Administration of the United States and thus can only be used when the benefit is greater than the risk ${ }^{1,11}$.

In summary, Doppler ultrasonography is recommended as an initial first-line test in pregnant women who are under clinical suspicion of DVT. If this study results positive, treatment ought to begin. In case of doubtful results, a venous MRI or venography with contrast should be performed as alternatives.

\section{PTE}

Early diagnosis of a pulmonary embolism during pregnancy is critical since there is a risk of a catastrophic maternal and fetal result if this is overlooked. If there is elevated clinical suspicion, empirical anticoagulation ought to be considered until the diagnostic process is complete. Similarly, a precise diagnosis is vital to avoid unnecessary treatment since this is linked to secondary effects in both the mother and the fetus.

Traditional clinical diagnostic keys of pulmonary embolism are dyspnea, tachycardia, tachypnea, pleuritic thoracic pain, and syncope. They are found in up to
Table 3. Wells score for PTE

\begin{tabular}{|l|c|}
\hline Clinical characteristics & Score \\
\hline Clinical symptoms of PTE & +3 \\
\hline Alternative diagnosis less probable than PTE & +3 \\
\hline Heart rate over 100 beats/min & +1.5 \\
\hline Immobilization or surgery within the past 4 weeks & +1.5 \\
\hline Previous PTE & +1.5 \\
\hline Hemoptysis & +1 \\
\hline Total score & \\
\hline
\end{tabular}

Interpretation of risk score (probability of PTE), $>6$ points, high risk $(78.4 \%)$, $2-6$ points, moderate risk $(27.8 \%),<2$ points, low risk (3.4\%), PTE: Pulmonary thromboembolism.

$90 \%$ of patients where a pulmonary embolus is shown. Nevertheless, these clinical signs and symptoms lack specificity and generate an ample differential diagnosis $^{13}$. The most common clinical signs and symptoms are an acute onset of symptoms, dyspnea, tachypnea, pleuritic thoracic pain, hemoptysis, syncope, tachycardia, cyanosis, pleural rub, and a fixed S2.

In the non-pregnant population, predictive clinical models such as the Wells score for Pulmonary Thormboembolism (PTE) (Table 3) stratify patients to establish the probability of having thrombotic events, according to which, confirmatory imaging diagnostic tests are conducted. It has a sensitivity of $100 \%$ and specificity of 90 when the score is higher or equal to 6 . However, it does not answer the questions among the pregnant population. Therefore, they ought to be taken to further studies $^{14}$.

Additional studies include DD; however, just as in those patients suspected of DVT, it is sensitive but not specific regarding pulmonary embolism. With a negative predictive value of $95 \%$, but only $25 \%$ specificity ${ }^{1,5}$, the study of arterial gases can reveal hypoxemia, hypocapnia, and respiratory alkalosis'.

Imaging studies such as Doppler ultrasonography show that approximately $70 \%$ of the patients with proven positron emission tomography (PET) have a proximal DVT ${ }^{1,5}$. A chest film has little specificity and sensitivity for PET diagnosis. It can show unspecified alterations of the lung, parenchyma, atelectasis, pleural spilling, cardiomegaly, an elevated ipsilateral hemidiaphragm, pulmonary artery enlargement, and wedge-shaped perfusion defects ${ }^{1,14}$. Pulmonary arteriography (PA) is considered the gold standard for PET diagnosis outside pregnancy, yet it is often used in high-risk cases which have negative compressive 
ultrasounds. A negative PA excludes clinically relevant PET. Angiography through MRI, which utilizes an injection of gadolinium, has a global sensitivity of only $77 \%$ compared to pulmonary angiography $(\mathrm{PA})^{15}$, and finally, helicoidal computed tomographic (CT) PA is not sensitive for the diagnosis of subsegmental clots. Thus, CT-PA can fail to exclude smaller peripheral lesions ${ }^{1}$.

An electrocardiogram can report a right bundle branch block, an axis displacement to the right, the presence of a $Q$ wave in derivations II, III, and aVF, an $S$ wave in derivations I and $\mathrm{aVL}>1.5 \mathrm{~mm}$, inversion of the $\mathrm{T}$ waves in derivations III and aVF, or the presence of auricular fibrillation'. The echocardiogram reports a hypokinetic and dilated right ventricle, tricuspid insufficiency, and dilation of the pulmonary artery, but it is useful only in $30-40 \%$ of the patients with PET when the transthoracic is performed and increases with the transesophageal echocardiogram, which has a sensitivity of $97 \%$ and a specificity between 88 and $100 \%$.

\section{DVT treatment in obstetrics}

Treatment should begin as soon as the DVT or PET diagnoses are made and, in some cases, treatment ought to begin with the suspicion even before confirming the diagnosis ${ }^{5}$. The initial steps in the treatment of pulmonary embolism are oxygen therapy, stabilization of blood pressure, and evolution of the cardiovascular and respiratory states of the patient $t^{1,5,7}$.

The main medical treatment is anticoagulation, and five different forms of treatment have been considered for the general population: Heparins, warfarin, surgery, vena cava filters, and thrombolytic therapy ${ }^{1,5,16}$.

\section{Heparins}

These include unfractioned heparin (UFH), low molecular weight heparin (LMWH), and factor Xa synthetic inhibitors (fondaparinux). All forms of heparin are administered through an injectable route, either subcutaneously or intravenously ${ }^{1,5,6}$.

Recommended schemes are as follows:

- Prophylactic UFH and subcutaneous (SC) UFH $5000 \mathrm{UI}$ dose every $12 \mathrm{~h}$.

- Adjusted-dose UFH, SC UFH every $12 \mathrm{~h}$ in adjusted doses until reaching an average interval in the partial time of thromboplastin within a therapeutic range (2-2.5 times the base value).

- Prophylactic LMWH, enoxaparin 40 mg SC every $24 \mathrm{~h}$, bemiparin at a dose of $3500 \mathrm{UI} \mathrm{SC}$ every $24 \mathrm{~h}$ or dalteparin at a dose of 5000 UISC every $24 \mathrm{~h}$.
Dosage of two or three medications may vary in the extremes of the body mass index values.

- Therapeutic LMWH at a dose adjusted to body weight, enoxaparin $1 \mathrm{mg} / \mathrm{kg}$ every $12 \mathrm{~h}$, dalteparin $200 \mathrm{U} / \mathrm{kg}$ every $12 \mathrm{~h}$, both subcutaneously.

\section{Warfarin}

It is an anticoagulant indicated for long-term use outside pregnancy, which has been linked with fetal defects. Embryopathy caused by warfarin is linked to exposure at 6-12 weeks of gestation and increases the risk of loss of pregnancy, anomalies in the central nervous system, and risk of fetal and neonatal hemorrhage during delivery due to fetal anticoagulation. Therefore, for women receiving prolonged anticoagulation therapy who become pregnant, the use of UFH or LMWH in place of warfarin is recommended. However, warfarin is indicated in patients with mechanical heart valves, due to its high risk of thrombosis, and in the postpartum period if necessary, because there is no significant excretion in breast milk ${ }^{17,18}$. When Vitamin $\mathrm{K}$ antagonists are used, an INR value of 2.0-3.0 should be achieved. At the beginning of the administration, it is administered simultaneously with hepatocyte nuclear factor or LMWH until they reach values in the INR $\geq 2.0$, and heparin is suspended.

\section{Inferior vena cava filters}

These are indicated when there are absolute contraindications for anticoagulant therapy (recent surgery, stroke, and active bleeding), adverse reactions or ineffectiveness of a previous anticoagulant (recurrent PET despite proper anticoagulation), or massive PET with significant compromise of the pulmonary vascular tree $e^{7,19}$.

\section{Surgery and thrombolytic therapy}

Surgical embolectomy ought to be reserved for those situations which endanger life. Massive PET with hemodynamic instability constitutes the only indication for thrombolytic therapy during pregnancy, given the high risk of causing a placental abruption ${ }^{5}$.

\section{General principles for VTE}

- Therapeutic anticoagulation should last between 12 and 20 weeks.

- Prophylactic anticoagulation should begin after initial treatment, for 6-12 weeks and until the patient reaches 6 weeks after delivery. 
- For complicated DVT, including those which affect iliofemoral vessels, prophylaxis for 4-6 weeks is recommended.

- Conversion to oral warfarin ought to be considered in the post-partum period, provided the patient accepts and complies with monitoring the level of medication.

- For patients with PET, prophylactic anticoagulation is recommended for 4-6 months.

- Maintain maternal partial pressure of oxygen above $70 \mathrm{~mm} \mathrm{Hg}$ or oxygen saturation $>94 \%$.

Among pregnant patients who have had a previous VTE during pregnancy, a risk of recurrence of $2.4-10 \%$ has been reported.

When establishing if a pregnant woman requires the use of antithrombotic prophylaxis, in case they were not in treatment already, three main points must be taken into consideration ${ }^{20,21}$.

- If the patient meets the criteria for prophylactic treatment, based on risk factors, concomitant pathologies, and pathological personal background.

- Risks and benefits of antithrombotic prophylaxis for the mother-fetus binomial.

- The mother's disposition toward the use of the indicated therapy.

Table 1 includes the criteria to establish which patients are at high risk of thromboembolic disease. If the patient meets at least one major criterion or two minor ones and requires an emergency $\mathrm{C}$-section, they are considered a candidate for antithrombotic prophylaxis since the risk of Venous Thromboembolism (VTE) increases 20,22-24.

Unfractionated heparin and LMWH constitute the two options recommended for prophylactic therapy in pregnant women. Compared to UFH, LMWH requires a single application a day, it does not require strict monitoring with laboratory work, and secondary effects such as thrombocytopenia induced by heparin, osteoporosis, and allergic reactions are fewer ${ }^{20,21}$.

Complementing the use of medications with anti-embolic averages or intermittent pneumatic compression is a recommended strategy for patients with a high risk of VTE who is in immediate surgical postpartum and in those patients where the risk factor for VTE persists during postpartum, maintaining anticoagulant up to 6 weeks after delivery ${ }^{25}$.

\section{References}

1. Rosenberg VA, Lockwood CJ. Thromboembolism in pregnancy. Obstet Gynecol Clin North Am. 2007;34:481-500, 10.

2. Segal JB, Streiff MB, Hofmann LV, Thornton K, Bass EB. Management of venous thromboembolism: a systematic review for a practice guideline. Ann Intern Med. 2007;146:211-22.

3. Gader AA, Haggaz AE, Adam I. Epidemiology of deep venous thrombosis during pregnancy and puerperium in sudanese women. Vasc Health Risk Manag. 2009;5:85-7.

4. James AH. Pregnancy and thrombotic risk. Crit Care Med. 2010;38:S57-63.

5. Greer IA. Thrombosis in pregnancy: updates in diagnosis and management. Hematol Am Soc Hematol Educ Program. 2012;2012:203-7.

6. James A, Committee on Practice Bulletins Obstetrics. Practice bulletin no. 123: thromboembolism in pregnancy. Obstet Gynecol. 2011;118: 718-29.

7. Jacobsen AF, Skjeldestad FE, Sandset PM. Incidence and risk patterns of venous thromboembolism in pregnancy and puerperium--a register-based case-control study. Am J Obstet Gynecol. 2008;198:233.e1-7.

8. Khan KS, Wojdyla D, Say L, Gülmezoglu AM, Van Look PF. WHO analysis of causes of maternal death: a systematic review. Lancet. 2006;367: 1066-74.

9. Casellas M, Fontcuberta J, Lecumberri R, et al. La Trombosis en el Embarazo y el Parto. $1^{\text {st }}$ ed. España: Leo Pharma; 2008. p. 5-29.

10. Chan WS, Spencer FA, Ginsberg JS. Anatomic distribution of deep vein thrombosis in pregnancy. CMAJ. 2010;182:657-60.

11. Chan WS, Lee A, Spencer FA, et al. D-dimer testing in pregnant patients: towards determining the next level in the diagnosis of deep vein thrombosis. J Thromb Haemost. 2010;8:1004-11.

12. Nijkeuter M, Ginsberg JS, Huisman MV. Diagnosis of deep vein thrombosis and pulmonary embolism in pregnancy: a systematic review. J Thromb Haemost. 2006;4:496-500.

13. Felludo $P$, Tapson V. The evaluation of suspected pulmonary embolism. N Engl J Med. 2003;349:1247-56.

14. Durán-Mendicuti A, Sodickson A. Imaging evaluation of the pregnant patient with suspected pulmonary embolism. Int J Obstet Anesth. 2011; 20:51-9.

15. Tan M, Huisman MV. The diagnostic management of acute venous thromboembolism during pregnancy: recent advancements and unresolved issues. Thromb Res. 2011;127 Suppl 3:S13-6.

16. Toyoda K. Antithrombotic therapy for pregnant women. Neurol Med Chir (Tokyo). 2013;53:526-30.

17. Ageno W, Gallus AS, Wittkowsky A, et al. Oral anticoagulant therapy. Chest. 2012;141 Suppl 2:e44S-88S.

18. Kearon $\mathrm{C}$, Kahn SR, Agnelli G, et al. Antithrombotic therapy for venous thromboembolic disease: American college of chest physicians evidence-based clinical practice guidelines ( $8^{\text {th }}$ edition). Chest. 2008;133: 454S-545S.

19. Valadares S, Serrano F, Torres R, Borges A. Inferior vena cava filter placement during pregnancy: an adjuvant option when medical therapy fails. Case Rep Obstet Gynecol. 2013;2013:821635.

20. Bates SM, Greer IA, Middeldorp S, et al. VTE, thrombophilia, antithrombotic therapy, and pregnancy: antithrombotic therapy and prevention of thrombosis, $9^{\text {th }}$ ed: American college of chest physicians evidence-based clinical practice guidelines. Chest. 2012;141:e691S-e736S.

21. Bain E, Wilson A, Tooher R, et al. Prophylaxis for venous thromboembolic disease in pregnancy and the early postnatal period. Cochrane Database Syst Rev. 2014;2:CD001689.

22. Jacobsen AF, Skjeldestad FE, Sandset PM. Ante and postnatal risk factors of venous thrombosis: a hospital-based case-control study. J Thromb Haemost. 2008;6:905-12.

23. Knight M, UKOSS. Antenatal pulmonary embolism: risk factors, management and outcomes. BJOG. 2008;115:453-61.

24. Voican I, Vladareanu PA, Onisai M, Nicolescu A, Vladareanu PR. Favorable outcome under anticoagulant therapy in a high risk pregnancy case report and short review of the (recent) literature. Maedica (Buchar). 2012; 7:339-43.

25. Royal College of Obstetricians and Gynaecologists. Reducing the Risk of Thrombosis and Embolism During Pregnancy and the Puerperium. RCOG Green-top Guideline; 2009. p. 1-35. 